\title{
Clinical characteristics of migraine in twelve sudanese families
}

\begin{abstract}
Introduction: Migraine headache is characterized by certain clinical features that were compiled according to certain criteria set by the international headache society (IHS) so as to provide a standardized definition, diagnosis and classification of headaches for both clinical practice and research studies
\end{abstract}

Aims: The aim of this study is to determine the clinical characteristics of migraine in Sudanese families and whether they adhere to international criteria set by the IHS

Patients and Methods: Following the description of the clinically diagnosed index case, a detailed validated questionnaire based on the IHS was distributed to all family members affected as well as non-affected after giving an informed consent. The responding family members were then classified as migraineurs and non-migraineurs after analysis of the questionnaire and confirmation of the diagnosis by two neurologists.

Results: From the 175 subjects who agreed to participate (from twelve pedigrees) 107 were migraineurs $(70.1 \%$ females, $29.9 \%$ males). Both migraine with aura (MA) and migraine without aura(MO) co-exist in the same family and even in the same individual with the most common migraine type being MO $(51.4 \%, \mathrm{~N}=55)$, followed by MA+MO $(32.7 \%$, $\mathrm{N}=35)$ and MA (15.9\%, $\mathrm{N}=17)$. Unilateral location, presence of nausea, photophobia and aggravation by exercise showed significant results (P value 0.000$)$.

Conclusions: The presentation of migraine headache showed results similar to that described by the HIS $2^{\text {nd }}$ edition in most of the criteria and differed in others. Co-morbidity with a number of diseases and migraine coexist.

Keywords: clinical characteristics, migraine with aura (ma), migraine without aura(mo), international headache society (ihs)
Volume 7 Issue 5 - 2017

\author{
M Salah ELmagzoub,' Sara M H Ibrahim,2 \\ Sami Fathi Abdalla ${ }^{3}$ \\ 'Department of physiology, National Ribat University, Sudan \\ ${ }^{2}$ Faculty of medicine, University of Gezira, Sudan \\ ${ }^{3}$ Almaarefa Colleges- Faculty of Medicine, Saudi Arabia
}

Correspondence: M Salah Elmagzoub, Faculty of medicine, National Ribat University, Khartoum, Sudan, Tel 00249-

912397699, Email msmagzoub@yahoo.com

Received: August 27, 2017 | Published: October 04, 2017

\section{Introduction}

The quality of life of a migraineur affects his/her familial and social situations, in addition to the impaired physical and emotional well being. ${ }^{1}$ In the Global Burden of Disease Survey 2010, migraine was ranked as the third most prevalent disorder and seventh-highest specific cause of disability worldwide. ${ }^{2}$ Migraine is a demanding and expensive disorder, beside the expensive medical costs there is a financial burden due to recurrent absenteeism from the work and hence diminished productivity. ${ }^{3}$

Migraine headache was known and described since ancient times. Hippocrates described the visual symptoms of migraine in $400 \mathrm{BC}$ as a shiny light, usually in the right eye, followed by severe pain beginning in the temples and eventually reaching the entire head and neck area. Hippocrates also noted the association between headache and various activities such as exercise. ${ }^{4}$

Yet, the first specific descriptions of variable types of headache were provided by Aretaeus of Cappadocia during the $1^{\text {st }}$ century A.D. Nevertheless it is Galen who used the term "hemicrania" to describe the unilateral pain sensation. ${ }^{6}$

A comprehensive and more detailed classification and diagnostic criteria however, were introduced by the International Headache Society (IHS) in 1988. ${ }^{7}$ In 1993, Blau (1993) had questioned the validity of the migraine criteria as patients may sometimes experience attacks that are difficult to categorize according to these criteria. ${ }^{8}$ This classification was then further revised and updated in 2004 ( $2^{\text {nd }}$ Edition) to include 14 types of head pain with migraine regarded as group one. Migraine as a primary head pain was further classified by the same classification into six subgroups, with migraine with aura
MA and migraine without aura MO being the most common types. ${ }^{9}$ The international headache society recently released a beta version of the third improved extensive criteria in 2013. ${ }^{10}$

Migraine has a strong familial aggregation with about $50 \%$ in the first degree relatives. Genetic contribution to migraine is diverse and subject to significant modification by environmental factors. ${ }^{11,12} \mathrm{Co}-$ morbidities of migraine and several other disorders have been reported in clinical series, case-control studies, and epidemiologic surveys. ${ }^{13}$ Cardiovascular, gastrointestinal, neurologic, and psychiatric systems and allergies as asthma are the most widely reported co-morbid disorders. ${ }^{14}$

As Migraine is a subjective disease, a detailed history is crucial in its diagnosis. The history, however, should be taken meticulously by an expert, together with a clinical examination performed by a neurologist. ${ }^{15}$ The diagnostic criteria set by the IHS have been the basis for migraine questionnaires'. ${ }^{16}$

In Sudan, Neurologists claim that migraine headache is very common but unfortunately no studies regarding the prevalence of migraine headache were done yet. Apart from the WHO data appeared in the Local Profil map, ${ }^{17}$ we could not find any published data concerning migraine. Therefore our current research intended to identify migraine profile using different parameters in order to have a clear picture of the disorder putting in mind the unique ethnic variations, the different cultural backgrounds, the consanguinity marriages and the diverse climatic changes in a vast country like ours. The present work aim to study the clinical characteristics of migraine in order to set the baseline that will further include a genetic, evoked potentials, neuroimaging and interleukins profile. 


\section{Materials and methods}

\section{Study design and Subjects}

The study was carried out in El Magzoub neuroscience centre and the faculty of medicine, National Ribat Teaching Hospital, between 2008 and 2010. One hundred seventy five subjects from twelve Sudanese families were included in this study. Family information was collected initially from the description of the clinically studied index case (proband) that was first seen at the neurology referred clinic. Following the description of the clinically diagnosed index case a detailed validated questionnaire based on the HIS ${ }^{4,6}$ was distributed to all family members affected as well as non-affected after giving an informed consent. The responding family members were then classified as migraineurs or non-migraineurs after analysis of the questionnaire and confirmation of the diagnosis by two neurologists. Only the data of migraineurs were included in this study.

\section{Data analysis}

Analysis of the clinical data and the questionnaire was carried out using STATA statistical package and Vassarstat online computational statistical software. P value $<0.05$ is considered significant. Approval for this study was given from ethical committee, university of Gezira. A written informed was signed by all participants.

\section{Results}

A cross sectional study that involved 175 subjects from 12 large Sudanese families; Afroasiatics (Ja'afra, Jaa'lia, Shawiga, Kwahla) and Nilosaharan (Halfaween and Mahas). 107 migraineurs were included in this study. Detailed history of the twelve pedigrees led to identification of 392 subjects. 189 (48.2\%) were migraineurs while 203 $(51.8 \%)$ were unaffected. Figure 1 shows one of the largest families. From the 175 who agreed to participate, 107 were migraineurs $(70.1 \%$ females, $29.9 \%$ males). Analysis of the questionnaire revealed that both migraine with aura and migraine without aura co-exist in the same family and even in the same individual with the most common migraine type being $\mathrm{MO}(51.4 \%, \mathrm{~N}=55)$, followed by $\mathrm{MA}+\mathrm{MO}$ $(32.7 \%, \mathrm{~N}=35)$ and MA $(15.9 \%, \mathrm{~N}=17)$ Figure 2.

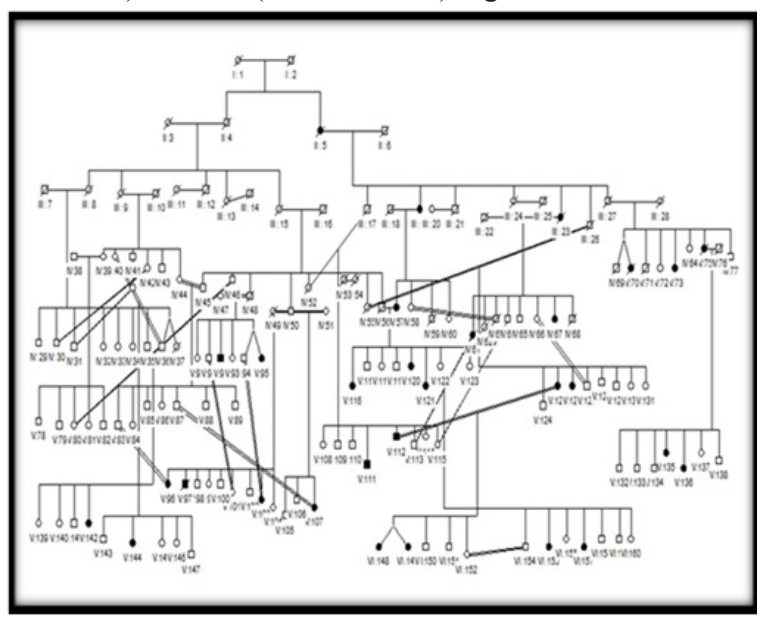

Figure I Family One ; circle is for female and square for male, Filled circle or square denotes subjects affected with migraine.

Types of migraine against gender and against duration of the attack are shown in Figures $3 \& 4$ respectively. Associated symptoms and triggering factors are shown in Figure 5. It should be noted that unilateral location, presence of nausea, photophobia and aggravation by exercise showed significant results ( $\mathrm{P}$ value 0.000$)$. Motor disorders, on the other hand, were not frequent even in MA (P value 0.23$)$. Only $4.8 \%$ of migraineurs $(\mathrm{N}=5)$ from different families experienced transient motor weakness typical of hemiplegic migraine once in their life.

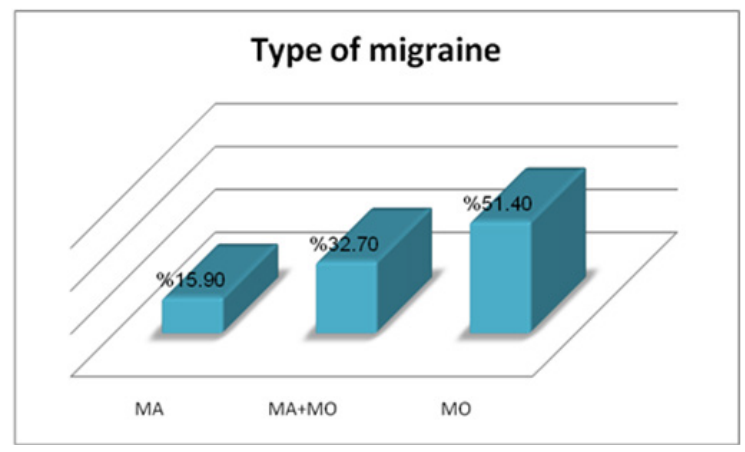

Figure 2 Types of migraine in the study group.

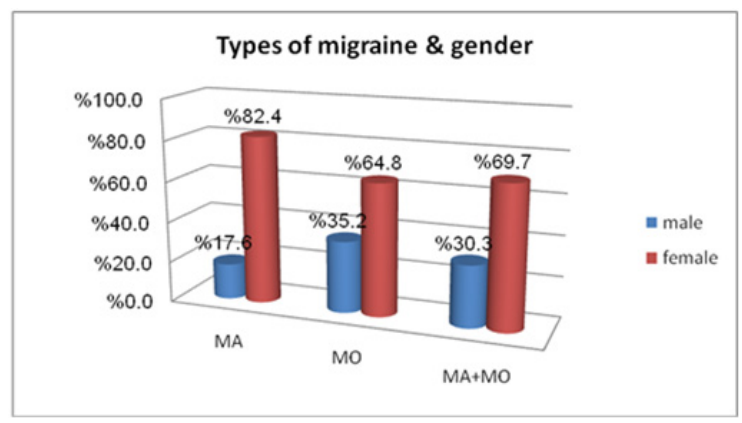

Figure 3 Types of migraine and gender.

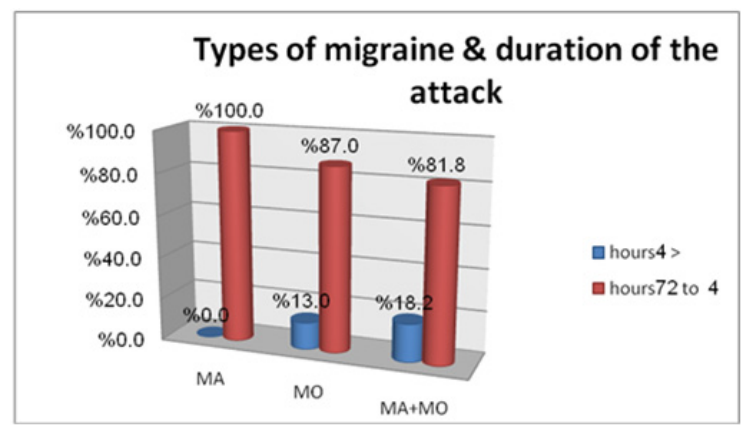

Figure 4 Types of migraine and duration of the attack.

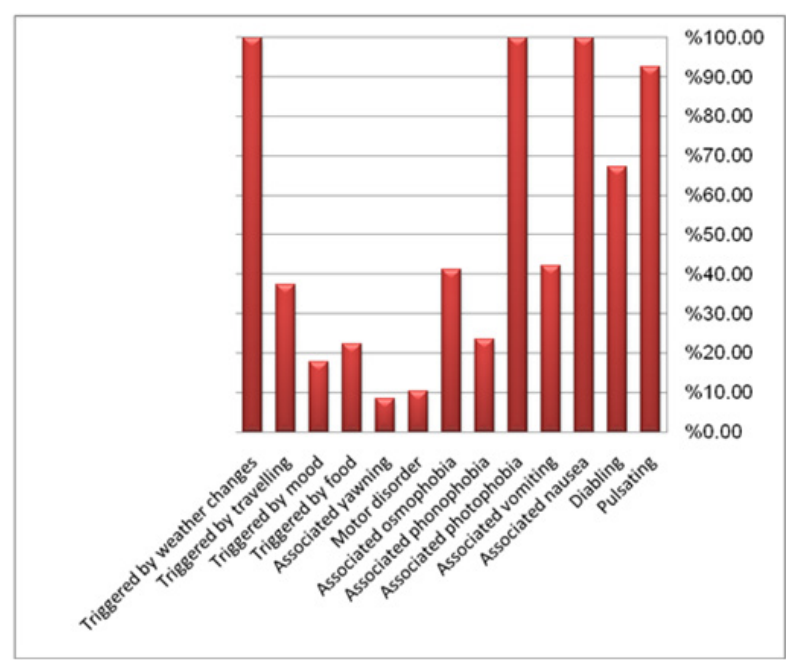

Figure 5 Associated symptoms and triggering factors of migraine in the study group. 
In migraine with aura patients, the most common aura symptoms are visual $(70 \%)$ followed by somatosensory $(23.4 \%)$ and motor $(6.5 \%)$. The most common co-morbid disorders with migraine within the twelve families were sinusitis $(100 \%)$ followed by hypertension $(91.7 \%)$, irritable bowel syndrome $(83.3 \%)$ asthma $(75 \%)$, and diabetes $(66.7 \%)$. Epilepsy, psychiatric disorders and depression were associated with migraine in about $50 \%$ of families.

Seventy percent of female migraineurs reported association between migraine and menstrual period, with $6.7 \%$ claimed cessation of headache after menopause. Four percent reported aggravation of headache and associated symptoms upon administration of contraceptive pills. Only one patient reported improvement of headache during pregnancy.

Regarding acute migraine treatment, only $23.4 \%$ of migraineurs claimed that they benefit from ergotamines while $88.7 \%$ use simple analgesics. Forty two percent use triptans for the severe attacks when simple analgesics were ineffective. Few migraineurs (14\%) use antiemetics when the attacks last longer than 48 hours and are accompanied by nausea and vomiting. Only $8.4 \%$ used preventive treatment which was discontinued after a short period of time. However, $32.7 \%$ of migraineurs prefer not to take any medication and choose to lie in a quite dim-lighted room instead.

\section{Discussion}

In the present study the presentation of migraine headache showed results similar to that described by the IHS (IHS,1988, IHS, 2004) ${ }^{4,6}$ in most of the criteria and differed in others. Unilateral location, duration of migraine, and aggravation of headache with minimum exercise all showed significant association with migraine, while disabling nature was not significantly associated. However migraine with aura and migraine without aura coexist in families and sometimes in the same individual. The most common type of migraine was $\mathrm{MO}$ accounting for more than $50 \%$ of cases.

Familial hemiplegic migraine was not reported and the discrete transient motor weakness that experienced by some migraineurs was sporadic and not recurrent. Some motor disorders like cervical pain and muscle spasms that usually accompany some migraine attack were reported in a number of the migraineurs (18.7\%).

Co-morbidity with several medical conditions like hypertension, asthma, epilepsy and depression coexist with migraine. The comorbidity with sinusitis renders some migraineurs to be underdiagnosed. This agreed with Schreiber et al who reported that about $90 \%$ of self-described sinus headache sufferers or physiciandiagnosed sinus headache patients have migraine. ${ }^{18}$ The hot climate and long autumn season in Sudan may explain the strong association between weather triggering and migraine. This finding is supported by J. Hoffmann et al who reported that the onset of an attack as well as high headache intensity was associated with lower temperature and higher humidity and that a subgroup of migraineurs is highly sensitive to changes of certain weather components. ${ }^{19}$ However, K Zebenholzer et al. ${ }^{20}$ concluded that the influence of weather factors on migraine and headache is small and questionable. ${ }^{20}$

Several studies reported that migraine has different presentations in different populations..$^{21,22}$ As reviewed by Landy approximately half of migraine sufferers have not received a diagnosis. ${ }^{23}$ In the present study, most of migraine patients are under-diagnosed and eventually undermanaged. This is in agreement to Dowson et al who reported that almost $90 \%$ of patients presenting to primary care practitioners with headache suffer from migraine. ${ }^{24}$
In Sudan, Neurologists claim that migraine headache is very common and that the severity of the attacks, associated nausea, vomiting, phonophobia, photophobia and the variable presentations of different attacks often render many patients to usually diagnose themselves as having sinus headaches, visual acuity disorder or even a toothache and thus wander around between different specialties like ENT surgeons, Ophthalmologists, Dentists, and even traditional healers before visiting a neurologist and be diagnosed as migraineurs.

Identification and perception of the different presentations of migraine by the caregivers and even by the migraineur himself and his worried family may lead to a better management. Thorough and meticulous descriptive classification and diagnostic criteria of migraine were introduced by the International Headache Society (IHS) in 1988. ${ }^{4}$ However, adherence to International Headache Society (IHS) criteria may lead to misdiagnosis of some types of headache. ${ }^{25}$ Accordingly, migraine presentations other than the typical unilateral throbbing headache with nausea and sensitivity to light and sound must be considered. ${ }^{26}$ Regardless of the minor differences, physicians currently diagnose migraine according to the criteria set by the IHS since these criteria represent the expert opinion of acknowledged clinicians on what is characteristic of migraine. Likewise IHS diagnostic criteria form the basis of most of recent researches on migraine. However, Nyholt et al,. ${ }^{27}$ \& Lea et al., ${ }^{28}$ had further dissected the common types of migraine according to latent class analysis (LCA). ${ }^{27,28}$ Another approach, trait component analysis (TCA), based on individual patient responses instead of clinical diagnoses was adopted by Antilla and colleagues in 2006 in order to classify patients into groups for genetic analysis. ${ }^{29}$

The present study is intended to form a basis for further research in familial migraine, yet it may serve as reference baseline data for even further research. As well the study can also aid the caregivers; neurologists, physicians and medical practitioners in clarifying the diagnosis and planning for a better management, regardless of the limited number of families included but they represent central Sudan with Afroasiatic or Nilosaharan origin.

It should be noted that the present study was carried out just before the launching of the beta version of the third revised criteria of the international headache society (ICHD-3 beta). Application of the latest criteria would definitely modify the results due to addition of new subtypes like chronic, probable and retinal migraine. The new criteria will at least justify the co existence of both migraine with aura and migraine without aura in the same individual. Therefore, a new questionnaire should be designed according to the final version in order to reclassify and reach a renewed diagnosis.

\section{Conclusion}

Migraine headache in Sudan has a presentation almost similar to that stated by the IHS. Migraine with aura and migraine without aura coexist in the same individual and same family. Co-morbidity of migraine coexists with a number of diseases in particular hypertension, epilepsy, sinusitis, asthma and stroke, a finding that needs further refining.

\section{Acknowledgements}

We gratefully acknowledge the assistance of the Sudanese ministry of higher education, for partially funding this research.

\section{Conflicts of Interest}

None. 


\section{Funding}

None.

\section{References}

1. Manack AN, Buse DC, Lipton RB. Chronic migraine: Epidemiology and disease burden. Curr Pain Headache Rep. 2011;15(1):70-78.

2. Steiner TJ, Birbeck GL, Jensen RH, et al. Headache disorders are third cause of disability worldwide. The Journal of Headache and Pain. $2015 ; 16: 58$

3. Vos T, Flaxman AD, Naghavi M. Years lived with disability (YLDs) for 1160 sequelae of 289 diseases and injuries 1990-2010: a systematic analysis for the Global Burden of Disease Study 2010. Lancet. 2010;380(9859):2163-2196

4. Lance JW, Goadsby PJ. Migraine: pathophysiology. In Mechanisms and Management of Headache. Oxford, Butterworth-Heinemann, UK. 1998. p.79-115.

5. Isler H. Retrospect: the history of thought about migrainefrom Areteus to 1920. In: Blau J(Ed.), Migraine: clinical, therapeutic, conceptual and research aspects. Chapman and Hall Medical, London, UK. 1987. p.659-674.

6. Pearce J. The ophthalmological complications of migraine. J Neurol Sci. 1968;6(1):73-81.

7. Classification and diagnostic criteria for headache disorders, cranial neuralgias and facial pain Headache Classification Committee of the International Headache Society. Cephalalgia. 1988; 8(Suppl 7):1-96.

8. Blau J N. Diagnosing migraine: are the criteria valid or invalid? Cephalalgia. 1993;13(12):21-24.

9. The International Classification of Headache Disorders: $2^{\text {nd }}$ edition. Cephalalgia. 2004;24(1):9-160.

10. The international classification of headache disorders, 3 rd edition (beta version). Cephalalgia. 2013;33(9):629-808.

11. Russell MB, Hilden J, Sorensen SA, ET AL. Familial occurrence of migraine without aura and migraine with aura. Neurology. 1993;43(7):1369-1373.

12. Kallela M, Wessman M, Farkkila M. Validation of a migraine-specific questionnaire for use in family studies. Eur J Neurol. 2001;8(1):61-66.

13. Merikangas K, Low N, Rasmussen B. Migraine comorbidity. In Migraine and other headache disorders. 2010.

14. Nancy cp Low, Kathleen Ries Merikangas. The comorbidity of migraine. CNS Spectrums. 2003; 8(6):433-444.
15. Olesen J. General principles of epidemiology. Discussion summary. In: Olesen J (Ed.), Headache Classification and Epidemiology. Raven Press, New York, USA. 1994. p.227-228.

16. Olesen J. Measurement techniques: questionnaire, telephone interview, clinical interview validation and reliability. In: Olesen J (Ed.) Headache Classification and Epidemiology. Raven Press, New York, USA. 1994. p.197-204.

17. Lokal profil. Disability-adjusted life year for migraines per 100,000 inhabitants in 2002. 2009.

18. Schreiber CHS, Powers C, Webster C, et al. Physician diagnosed and patient self-described sinus headache is predominantly migraine. Proc Am Headache. 2002.

19. J Hoffmann, H Lo, L Neeb, et al. Weather sensitivity in migraineurs. Journal of Neurology. 2011; 258(4):596-602.

20. K Zebenholzer, E Rudel, S Frantal. Migraine and weather: a prospective diary-based analysis. Cephalalgia. 2011;31(4):391-400.

21. Nicholson RA, Rooney M, Vo K, et al. Migraine care among different ethnicities: do disparities exist? Headache. 2006;46(5):754-765.

22. Jorge Matias-Guiu, Cristina Fernandez, Jesus Porta-Etessam, et al Factors Associated with the Differences in Migraine Prevalence Rates between Spanish Regions. The Scientific World Journal. 2014. p. 6.

23. Lipton RB, Diamond S, Reed M, et al. Migraine diagnosis and treatment: results from the American Migraine Study II. Headache. 2001;41(7):638-645

24. Dowson A, D C, Tepper S, Newman L, et al. The prevalence and diagnosis of migraine in a primary care setting-insights from the Landmark Study. In 14th Migraine Trust Meeting, London. 2002.

25. Kaniecki RG. Migraine and tension-type headache: an assessment of challenges in diagnosis. Neurology. 2002;58(9 Suppl 6):S15-S20.

26. Landy S, Savani N, Shackelford S, et al. Efficacy and tolerability of sumatriptan tablets administered during the mild-pain phase of menstrually associated migraine. Int J Clin Pract. 2004;58(10):913-919.

27. Nyholt DR, Morley KI, Ferreira MA, et al. Genomewide significant linkage to migrainous headache on chromosome $5 \mathrm{q} 21$. AmJ HumGenet. 2005;77(3):500-512.

28. Lea RA, Ovcaric M, Sundholm J, et al. The methylenetetrahydrofolate reductase gene variant $\mathrm{C} 677 \mathrm{~T}$ influences susceptibility to migraine with aura. BMC Med. 2004;2:3.

29. Anttila V, Kallela M, Oswell G, et al. Trait components provide tools to dissect the genetic susceptibility of migraine. Am J Hum Genet. 2006;79(1):85-99. 